\title{
Catheter ablation in atrial fibrillation: a state-of-the-art review
}

This article was published in the following Dove Press journal:

Research Reports in Clinical Cardiology

23 November 2015

Number of times this article has been viewed

\section{Antonio Sorgente' \\ Riccardo Cappato ${ }^{2}$}

'Heart and Vascular Institute, Cleveland Clinic Abu Dhabi, Abu Dhabi, United Arab Emirates;

${ }^{2}$ Arrhythmia and Electrophysiology Research Center, IRCCS Humanitas Clinical and Research Center, Rozzano, Milan, Italy

\section{Video abstract}

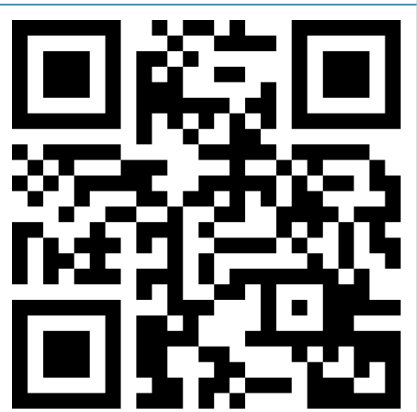

Point your SmartPhone at the code above. If you have a QR code reader the video abstract will appear. Or use: http://youtu.be/fDcpurqxeBM
Correspondence: Riccardo Cappato Arrhythmia and Electrophysiology Research Center, IRCCS Humanitas Clinical and Research Center, Via Alessandro Manzoni 56, 20089 Rozzano, Milan, Italy

Email riccardo.cappato@humanitas.it
Abstract: There are no doubts that paroxysmal atrial fibrillation is driven by triggers located at the ostia of pulmonary veins. Cardiac electrophysiologists have to thank the seminal work of Dr Haissaguerre in demonstrating for the first time this finding in human hearts in 1998. On this premise, atrial fibrillation ablation performed through pulmonary vein electrical isolation has become a mainstay of the non-pharmacological treatment of this arrhythmia. The scope of this brief and concise review is to provide a state-of-the-art of catheter ablation of atrial fibrillation, considering the obvious different outcomes in different varieties of atrial fibrillation. If the results in the treatment of paroxysmal atrial fibrillation are really promising, it is clear that more research and more clinical trials are warranted for the cure of non-paroxysmal atrial fibrillation, which cause seems to be more multifactorial and less dependent on electrical triggers.

Keywords: atrial fibrillation, arrhythmia, sinus rhythm, pulmonary veins, anticoagulation, catheter ablation

\section{Introduction}

Atrial fibrillation (AF) is the most common cardiac arrhythmia encountered in clinical practice. ${ }^{1}$ The therapeutic approach toward this arrhythmia usually comprises initiation of anticoagulation therapy and management of the heart rate through rhythm or rate control strategies. With regard to rhythm control, the high recurrence rate linked to the use of antiarrhythmic drugs (AADs) has prompted the development of alternative nonpharmacologic options. Electrical cardioversion, which represents the easiest and most accessible nonpharmacological strategy for persistent AF, does not affect the natural history of this arrhythmia. This has therefore favored the spread of catheter ablation of $\mathrm{AF}$ as the most valid treatment option in patients with drug-refractory $\mathrm{AF}^{1}$ who desire to be more consistently and steadily reverted into sinus rhythm.

\section{Results in paroxysmal AF}

The rationale behind catheter ablation stems from the finding that AF can be triggered from electrical impulses generated inside one or more pulmonary veins. ${ }^{2,3}$ Pulmonary veins have muscular sleeves which extend into the left atrium and help regulate blood flow toward the left cardiac chambers. Histopathological observations allowed the discovery of P cells, transitional cells, and Purkinje cells in these myocardial muscle extensions. These observations combine perfectly with the hypothesis that pulmonary veins can be the source of rapid electrical activity triggering $\mathrm{AF}^{3,4}$

Several studies have shown that electrical isolation of pulmonary veins achieved with various energy sources (ie, radiofrequency, cryothermal, laser energy) significantly 
reduces the burden of AF in patients affected by this arrhythmia as compared to AAD treatment. ${ }^{4,5}$ Catheter ablation has proven mostly effective in patients with paroxysmal AF. In these patients, triggers account for the most common precipitating cause of AF, whereas other mechanisms are also responsible in nonparoxysmal AF. Piccini et $\mathrm{al}^{6}$ have conducted a specific meta-analysis to test the general hypothesis that pulmonary vein isolation is more efficacious than AADs in preventing recurrences of AF. The primary endpoint tested in this study was freedom from recurrent $\mathrm{AF}$ at 12 months follow-up. Using a random-effects modeling according to intention to treat, aggregate data from six randomized clinical trials (RCTs) totaling 693 patients were analyzed. Pulmonary vein isolation was associated with significantly increased odds of freedom from AF at 12 months of follow-up (77\% vs 29\%; odds ratio, 9.74; 95\% CI [confidence interval], 3.98-23.87). After elimination of those studies which enrolled patients with persistent AF, the probability of maintaining sinus rhythm was even greater (odds ratio, 15.78; 95\% CI, 10.07-24.73). The authors also found a reduction in hospitalizations for cardiovascular causes (14 vs 93 per 100 person-years; rate ratio, 0.15 ; 95\% CI, 0.10-0.23). Importantly, $17 \%$ of the patients who underwent a catheter ablation needed a repeat procedure within the 12-month follow-up. The rate of major complications in the invasive arm was $2.6 \%$ (similar to what was previously reported for other invasive procedures) against a rate of reported adverse events associated with AAD therapy of $8 \%$. These data were corroborated by those from another meta-analysis by Calkins et al, ${ }^{7}$ which included randomized and nonrandomized clinical trials. During 14 months follow-up, the overall success rate was $52 \%(95 \% \mathrm{CI}, 47 \%-57 \%)$ for patients on AADs and $57 \%(95 \% \mathrm{CI}, 50 \%-64 \%)$ for patients receiving a single procedure of catheter ablation of AF. The success rate after ablation rose to $71 \%(95 \% \mathrm{CI}, 65 \%-77 \%)$ after administration of previously ineffective AADs.

Overall, pulmonary vein isolation has proven effective for the treatment of patients with paroxysmal AF. Improvements are awaited in order to prolong the efficacy of AF ablation and reduce the recurrence of any atrial arrhythmia over time. In fact, several studies have shown that pulmonary vein isolation can be transient in a significant proportion of patients. Three of the most important RCTs on this topic (Thermocool AF trial, ${ }^{8}$ A4 study, ${ }^{9}$ and RAAFT- $2^{10}$ ) reported a nonnegligible rate of repeat procedures $(12.6 \%-43.4 \%)$, with conduction recovery across acutely isolated pulmonary veins being the most common condition associated with AF recurrence. Among the theories that have been proposed to explain conduction recovery, acute formation of tissue edema during the index procedure and incomplete lines of transmural ablation are the most accepted ones. ${ }^{11}$ Recovery of tissue edema after radiofrequency ablation is usually linked to poor contact of the ablation catheter at the endocardial side. It has been clearly demonstrated that a rough quantification of tissue contact is obtainable indirectly from electrograms amplitude and impedance values, but this information is not always accurate, even when it comes from expert operators. The recent introduction of contact force-sensing technology has provided investigators with a new technology to test the possibility of overcoming this limitation. The EFFICAS-1 trial $^{12}$ showed that applying a catheter contact force of $20 \times g$ with a minimum of $400 \times \mathrm{g} / \mathrm{s}$ force-time integral is associated with a durable lesion, as accounted by the number of recurrences of atrial arrhythmias and the location of gaps and electrical reconnections evidenced during follow-up procedures. Accurate information about the force applied to the catheters also allows to consider a reduced probability of perforation.

The efficacy of radiofrequency or cryothermal energy delivery on pulmonary vein isolation has also been assessed using intravenous administration of adenosine, following acute pulmonary vein isolation. After pulmonary vein isolation, ${ }^{13,14}$ adenosine administration may unmask electrical reconnection of the pulmonary veins (the so-called "awakening" of dormant pulmonary vein conduction) and can guide the operator to identify those gaps accordingly with further energy applications at sites of reconnection. A nonrandomized, single center study which has used adenosine to guide pulmonary vein isolation could demonstrate that this approach can reduce repeat-procedure rates by nearly $50 \%$; a multicenter randomized study, ADVICE (ADenosine following pulmonary Vein Isolation to target dormant Conduction Elimination), ${ }^{15}$ will shed further light on the topic.

The completeness of pulmonary vein isolation can be further investigated using pace mapping along the line of antral isolation. According to data from a recent study, ${ }^{16}$ adding further radiofrequency pulses along the ablation line at gap sites showing residual conduction as elicited by selected pacing on pulmonary vein antrum is reliable and is associated with an improved outcome. After a mean follow-up of 18 months, patients in the paced group had presented with an $18 \%$ recurrence rate as compared to the conventional group, who experienced $48 \%$ of atrial arrhythmia recurrences $(P=0.001)$.

An alternative strategy to improve catheter-tissue contact is represented by the development of balloon-based catheter technologies. The advantages of using a balloon device stems 
in the unique possibility that these devices offer to deploy a circumferential "single-shot" lesion within the antrum of pulmonary veins using cryothermal energy. The FIRE and ICE study ${ }^{17}$ will assess the efficacy and the safety of cryothermal energy in comparison to radiofrequency energy and will provide objective data to guide selection and usage of ablation catheters in the treatment of AF.

\section{Results in nonparoxysmal AF}

Pulmonary vein isolation is a consolidated technique to address drug-refractory paroxysmal AF. The results of this technique in patients with nonparoxysmal AF are less consistent. ${ }^{1}$ As a consequence, several alternative options have been proposed in clinical practice in addition to pulmonary vein isolation, most often in the absence of a clear pathophysiological rationale. One of these options is complex fractioned atrial electrograms (CFAEs), introduced by Nademanee et al. ${ }^{18}$ In these authors' definition, CFAEs represent "low voltage atrial electrograms (ranging from 0.04 to $0.25 \mathrm{mV}$ ) that have fractionated electrograms composed of two or more deflections, and/or have a perturbation of the baseline with continuous deflection of a prolonged activation complex". Nademanee et $\mathrm{al}^{18}$ demonstrated that ablation of CFAE was efficacious in restoring sinus rhythm in patients with both paroxysmal and nonparoxysmal AF, with $87 \%$ of them free of atrial arrhythmias recurrence at an average follow-up of $839 \pm 493$ days. Henceforth, this ablation technique has been applied in a number of clinical studies, generally, in addition to pulmonary vein isolation. The results of CFAE ablation have been stressed in a recent meta-analysis by Wu et al. ${ }^{19}$ Unifying the data coming from eleven studies, which share additional CFAE ablation as a common technique to treat patients with both paroxysmal and nonparoxysmal AF, these authors demonstrated that CFAE ablation, in addition to pulmonary vein isolation, reduces recurrence of atrial tachyarrhythmias after a single procedure (pooled RR [relative risk], 0.73; 95\% CI, 0.61-0.88; $P=0.0007$ ) at $\geq 3$-month follow-up compared to pulmonary vein isolation alone. More in particular, additional CFAE ablation reduced recurrence rate in nonparoxysmal AF (RR, $0.68 ; 95 \%$ CI, 0.47-0.99; $P=0.05)$, without any significant effect on patients with paroxysmal AF (RR, 0.79; 95\% CI, $0.59-1.06 ; P=0.12$ ). This partial benefit in terms of recurrence of nonparoxysmal AF was counterbalanced by a higher incidence of postprocedural atrial tachycardias (RR, 1.77; 95\% CI, 1.02-3.07; $P=0.04)$, increased mean procedural times $(245.4+75.7$ vs $189.5+62.3$ minutes, $P<0.001)$, mean fluoroscopy $(72.1+25.6$ vs $59.5+19.3$ minutes, $P<0.001)$, and mean RF energy application times $(75.3+38.6$ vs $53.2+27.5$ minutes, $P<0.001)$. Very recently, Verma et $\mathrm{al}^{20}$ have tried to figure out if a more selective approach in CFAE ablation (ie, CFAE showing a continuous electrical activity and absence of isoelectric segments for more than $75 \%$ of their duration) can reduce the length of these procedures and impact the outcome in terms of atrial arrhythmias recurrence. This selective approach reduced fluoroscopy times resulting in a similar incidence of acute AF termination, but unfortunately, at 1-year follow-up, freedom from AF/atrial flutter/ atrial tachycardia recurrence and need of a repeat procedure were significantly higher than in patients treated with the traditional and generalized CFAE ablation.

An innovative curative approach to nonparoxysmal AF has recently been proposed by Narayan et al, ${ }^{21}$ who hypothesized that ablation of AF substrates (the so-called electrical rotors or focal sources) with the help of a special three dimensional electroanatomical mapping system can ensure better and longer arrhythmia-free survival in these patients than ablation of pulmonary and nonpulmonary veins triggers. The CONFIRM (Conventional Ablation for Atrial Fibrillation With or Without Focal Impulse and Rotor Modulation) $)^{22}$ trial enrolled 92 patients with high-burden paroxysmal AF or persistent $\mathrm{AF}$ and randomized them in a two-arm 1:2 design, by ablation at sources (Focal Impulse and Rotor Modulation [FIRM]-guided) followed by conventional ablation, mainly pulmonary vein isolation $(n=36)$, or conventional ablation alone ( $\mathrm{n}=71$; FIRM-blinded). AF termination or slowing of its average cycle length was obtained in about $85 \%$ of patients in the active arm whereas this objective was reached only in $20 \%$ of FIRM-blinded patients. Interestingly, ablation of sources of AF in addition to pulmonary vein isolation ensured a better outcome at a median follow-up of 273 days (interquartile range: 132-681 days) after a single procedure $(82.4 \%$ vs $44.9 \% ; P<0.001)$. No differences in adverse events between groups were reported. Recently, a continuation of the follow-up on the same patients to about 3 years led to another publication by the same group of authors: superiority of rotors ablation in addition to pulmonary vein isolation was confirmed after a median follow-up of 890 days (interquartile range: $224-1,563$ days), both in terms of AF and of atrial arrhythmias recurrences. Even if the results of these studies are encouraging, a drawback of this ablation technique is that it needs a special electroanatomical mapping system, and therefore, time and training would be needed before getting more consistent and reliable data.

Haissaguerre et $\mathrm{al}^{23}$ have also tried to understand the pathophysiology of human nonparoxysmal AF and to 
investigate if termination of AF could be considered a reliable target for catheter ablation in these subset of patients. The main scope of their research was to tailor the ablation procedure and strategy to the patient, assuming that AF can hide different pathophysiological mechanisms, even in the same patient. First, they evaluated if noninvasive mapping of AF through a commercially available system (ECVUE, CardioInsight Technologies Inc., Cleveland, OH, USA) could identify driver domains, and if duration of AF could affect their distribution and their quantity through the atria. The second objective of their research was to understand if this mapping information could modify the quantity of radiofrequency energy given to obtain intraprocedural termination of AF, using as control a matched group of patients treated previously by the use of the conventional ablation technique. Even if AF electroanatomical maps showed a great variability in terms of temporal behavior of driver activities (which evidenced a high degree of instability), these drivers recurred consistently in the same regions across the atria (with a clear predominance of the left atrium over the right) and seemed to share more a reentrant mechanism than a focal one ( $80.5 \%$ vs $19.5 \%)$. Ablation of these drivers terminated $75 \%$ and $15 \%$ of persistent and longlasting $\mathrm{AF}$, respectively, with termination rate falling down dramatically when AF duration was longer than 6 months. The results of follow-up were in line with those previously obtained by the same group with a stepwise ablation approach: at 12 months, patients with AF termination were free from $\mathrm{AF}$, similar to the control population $(87 \% ; P=$ not significant). The population of this study was relatively young (mean age, 59 years), predominantly males $(82 \%)$, with long lasting AF (>12 months) accounting only for about $20 \%$ of cases. Left atrial anteroposterior diameter was $48 \mathrm{~mm}$. Almost a half of these patients were treated with amiodarone before the ablation procedure, and about $20 \%$ had already undergone pulmonary vein isolation. This means that the results of their study cannot be applied on the vast majority of patients with persistent or permanent AF, who are usually older and with more dilated atria. Further investigations are warranted to understand if the results of this study can be applied also on a wider population. Furthermore, it has to be considered that this procedure needs a sophisticated mapping system, which can display its application on a larger scale, especially in nonacademic environments or in countries with economical constraints.

\section{Conclusion}

In conclusion, catheter ablation of AF is an established treatment option for drug-refractory AF. Pulmonary vein isolation is the treatment of choice for patients with paroxysmal
AF. Patients with nonparoxysmal AF benefit only partially from pulmonary vein isolation, but encouraging results have been obtained recently with alternative strategies of ablation. Further studies are warranted to deepen our knowledge on the mechanisms that stand behind AF and to obtain longerterm results of new ablation techniques for this complex and common arrhythmia.

\section{Disclosure}

The authors report no conflicts of interest in this work.

\section{References}

1. Calkins H, Kuck KH, Cappato R, et al. 2012 HRS/EHRA/ECAS Expert Consensus Statement on Catheter and Surgical Ablation of Atrial Fibrillation: recommendations for patient selection, procedural techniques, patient management and follow-up, definitions, endpoints, and research trial design. Europace. 2012;14(4):528-606.

2. Haïssaguerre M, Jaïs P, Shah DC, et al. Spontaneous initiation of atrial fibrillation by ectopic beats originating in the pulmonary veins. $N \mathrm{Engl}$ J Med. 1998;339(10):659-666.

3. Jaïs P, Haïssaguerre M, Shah DC, et al. A focal source of atrial fibrillation treated by discrete radiofrequency ablation. Circulation. 1997; 95(3):572-576.

4. Sorgente A, Chierchia GB, Capulzini L, et al. Atrial fibrillation ablation: a single center comparison between remote magnetic navigation, cryoballoon and conventional manual pulmonary vein isolation. Indian Pacing Electrophysiol J. 2010;10(11):486-495.

5. Xu J, Huang Y, Cai H, et al. Is cryoballoon ablation preferable to radiofrequency ablation for treatment of atrial fibrillation by pulmonary vein isolation? A meta-analysis. PLoS One. 2014;9(2):e90323.

6. Piccini JP, Lopes RD, Kong MH, Hasselblad V, Jackson K, Al-Khatib SM. Pulmonary vein isolation for the maintenance of sinus rhythm in patients with atrial fibrillation: a meta-analysis of randomized, controlled trials. Circ Arrhythm Electrophysiol. 2009;2(6):626-633.

7. Calkins H, Reynolds MR, Spector P, et al. Treatment of atrial fibrillation with antiarrhythmic drugs or radiofrequency ablation: two systematic literature reviews and meta-analyses. Circ Arrhythm Electrophysiol. 2009;2(4):349-361.

8. Wilber DJ, Pappone C, Neuzil P, et al; ThermoCool AF Trial Investigators. Comparison of antiarrhythmic drug therapy and radiofrequency catheter ablation in patients with paroxysmal atrial fibrillation: a randomizedcontrolled trial. JAMA. 2010;303:333-340.

9. Jaïs P, Cauchemez B, Macle L, et al. Catheter ablation versus antiarrhythmic drugs for atrial fibrillation: the A4 study. Circulation. 2008;118:2498-2505.

10. Morillo CA, Verma A, Connolly SJ, et al; RAAFT-2 Investigators. Radiofrequency ablation vs antiarrhythmic drug sas first-line treatment of paroxysmal atrial fibrillation (RAAFT-2): a randomized trial. JAMA. 2014;311:692-700.

11. Cheema A, Dong J, Dalal D, et al. Incidence and time course of early recovery of pulmonary vein conduction after catheter ablation of atrial fibrillation. J Cardiovasc Electrophysiol. 2007;18:387-391.

12. Neuzil P, Reddy VY, Kautzner J, et al. Electrical reconnection after pulmonary vein isolation is contingent on contact force during initial treatment: results from the EFFICAS I study. Circ Arrhythm Electrophysiol. 2013;6(2):327-333.

13. Arentz T, Macle L, Kalusche D, et al. "Dormant" pulmonary vein conduction revealed by adenosine after ostial radiofrequency catheter ablation. J Cardiovasc Electrophysiol. 2004;15:1041-1047.

14. Tritto M, De Ponti R, Salerno-Uriarte JA, et al. Adenosine restores atrio-venous conduction after apparently successful ostial isolation of the pulmonary veins. Eur Heart J. 2004;25(23):2155-2163. 
15. Macle L, Khairy P, Verma A, et al; ADVICE Study Investigators. Adenosine following pulmonary vein isolation to target dormant conduction elimination (ADVICE): methods and rationale. Can J Cardiol. 2012;28(2):184-190.

16. Steven D, Sultan A, Reddy V, et al. Benefit of pulmonary vein isolation guided by loss of pace capture on the ablation line: results from a prospective 2-center randomized trial. J Am Coll Cardiol. 2013;62(1):44-50.

17. Fürnkranz A, Brugada J, Albenque JP, et al. Rationale and design of FIRE AND ICE: a multicenter randomized trial comparing efficacy and safety of pulmonary vein isolation using a cryoballoon versus radiofrequency ablation with 3D-reconstruction. J Cardiovasc Electrophysiol. 2014;25(12):1314-1320.

18. Nademanee K, Schwab MC, Kosar EM, et al. Clinical outcomes of catheter substrate ablation for high-risk patients with atrial fibrillation. J Am Coll Cardiol. 2008;51:843-849.

19. Wu SH, Jiang WF, Gu J, et al. Benefits and risks of additional ablation of complex fractionated atrial electrograms for patients with atrial fibrillation: a systematic review and meta-analysis. Int J Cardiol. 2013 169(1):35-43.
20. Verma A, Sanders P, Champagne J, et al. Selective complex fractionated atrial electrograms targeting for atrial fibrillation study (SELECT AF): a multicenter, randomized trial. Circ Arrhythm Electrophysiol. 2014;7(1):55-62.

21. Narayan SM, Baykaner T, Clopton P, et al. Ablation of rotor and focal sources reduces late recurrence of atrial fibrillation compared with trigger ablation alone: extended follow-up of the CONFIRM trial (Conventional Ablation for Atrial Fibrillation With or Without Focal Impulse and Rotor Modulation). J Am Coll Cardiol. 2014;63(17):1761-1768.

22. Narayan SM, Krummen DE, Shivkumar K, Clopton P, Rappel WJ, Miller JM. Treatment of atrial fibrillation by the ablation of localized sources: CONFIRM (Conventional Ablation for Atrial Fibrillation With or Without Focal Impulse and Rotor Modulation) trial. J Am Coll Cardiol. 2012;60(7):628-636.

23. Haissaguerre M, Hocini M, Denis A, et al. Driver domains in persistent atrial fibrillation. Circulation. 2014;130(7):530-538.
Research Reports in Clinical Cardiology

\section{Publish your work in this journal}

Research Reports in Clinical Cardiology is an international, peerreviewed, open access journal publishing original research, reports, editorials, reviews and commentaries on all areas of cardiology in the clinic and laboratory. The manuscript management system is completely online and includes a very quick and fair peer-review system.

\section{Dovepress}

Visit http://www.dovepress.com/testimonials.php to read real quotes from published authors. 